\title{
Guía de Práctica Clínica: Actualización y combinación de las guías de cuidados preventivos de la fuerza de tareas de EE.UU. y Canadá (Tercera parte)
}

Combination and update of preventive care guidelines of the U.S. and Canadian task forces

Agustín Ciappon

\begin{abstract}
Resumen
La Fuerza de Tareas Preventiva de los EE.UU. (sigla en inglés: USPSTF) y la Fuerza de Tareas Canadiense de Cuidados Preventivos de la Salud (sigla en inglés: CTFPHC) son las dos instituciones más importantes del mundo en la evaluación de cuidados preventivos. Ambas emiten y actualizan constantemente recomendaciones preventivas; y las mismas pueden accederse en forma completa y gratuita a través de sus sitios Web (http://www.ahrq.gov/clinic/cps3dix.htm, y http://www.ctfphc.org). En esta tercera entrega continuamos con la actualización de prácticas referidas al rastreo preventivo de enfermedades de transmisión sexual y su justificación clínica emitida por ambas entidades. No se incluyen en esta revisión prácticas como inmunizaciones ni de profilaxis postexposición de enfermedades infecciosas, pues estas se rigen habitualmente por normas locales como las Normas Nacionales de Vacunación. Muchas recomendaciones emitidas hace varios años se encuentran bajo revisión pero las mantenemos a título informativo hasta que los cambios sean confirmados.
\end{abstract}

\section{Abstract}

The US Preventive Services Task Force (USPSTF) and the Canadian Task Force on Preventive Health Care (CTFPHC) are the two most important institutions in the world in the evaluation of preventive care. Both released and updated preventive recommendations, which can be accessed in full and free through its Web sites (http://www.ahrq.gov/clinic/cps3dix.htm and http://www.ctfphc.org ). In this third issue we continue to update on preventive screening of sexually transmitted diseases and its clinical justification issued by both entities. Practices as immunizations and post exposure prophylaxis of infectious diseases are not included in this review, as these are usually governed by local rules. Many recommendations made several years ago are under review but we keep them until the changes are confirmed.

Palabras clave: guía de práctica clínica, rastreo preventivo, enfermedades de transmisión sexual. Key words: clinical practice guideline, preventive screening, sexually transmitted diseases.

Ciapponi A. Actualización y combinación de las guías de cuidados preventivos de las fuerzas de tareas de EE.UU. y Canadá (tercera parte). Evid. Act. Pract. Ambul. 12(4). 146-149. Oct-Dic. 2009

Las principales recomendaciones referidas a la prevención de enfermedades de transmisión sexual (ETS) se resumen en la tabla 1.

Tabla 1: recomendaciones para la prevención de enfermedades de transmisión sexual.

\begin{tabular}{|c|c|c|c|c|c|}
\hline Problema & Población diana & Estrategia de rastreo & \multicolumn{2}{|c|}{ Recomendación } & Publicación \\
\hline \multirow[t]{2}{*}{ Sífilis } & Embarazadas y personas de alto riesgo & \multirow[t]{2}{*}{ VDRL } & A & $\mathrm{I} \oplus \oplus \oplus \oplus$ & $5 / 2009$ \\
\hline & Personas de bajo riesgo & & D & $\mathrm{I} \oplus \oplus \oplus \oplus^{\#}$ & $7 / 2004$ \\
\hline \multirow[t]{3}{*}{ Gonorrea } & Mujeres de alto riesgo & \multirow{3}{*}{$\begin{array}{c}\text { Examen directo y } \\
\text { cultivo } \\
\text { endocervical o uretral }\end{array}$} & $B$ & $\mathrm{II} \oplus \oplus \oplus \mathrm{O}$ & \multirow{3}{*}{$5 / 2005$} \\
\hline & Embarazadas u hombres de alto riesgo & & $I^{\star}$ & $-\oplus \oplus \# \mathrm{OO}$ & \\
\hline & \begin{tabular}{|l|} 
Población general adulta \\
\end{tabular} & & $\mathrm{D}$ & $\mathrm{I} \oplus \oplus \oplus \oplus^{\#}$ & \\
\hline \multirow[t]{2}{*}{ Hepatitis B } & Embarazadas & \multirow{2}{*}{$\begin{array}{r}\text { Serología } \\
\text { (HBsAg) }\end{array}$} & A & $\mathrm{I} \oplus \oplus \oplus \oplus$ & $6 / 2009$ \\
\hline & \begin{tabular}{|l|} 
Población general \\
\end{tabular} & & $\mathrm{D}$ & $\mathrm{I} \oplus \oplus \oplus \oplus^{\#}$ & $2 / 2004$ \\
\hline \multirow[t]{2}{*}{ Hepatitis C } & Población general & \multirow[t]{2}{*}{ Serología } & D & $\mathrm{I} \oplus \oplus \oplus \oplus^{*}-$ & \multirow[t]{2}{*}{$3 / 2004$} \\
\hline & Adultos de alto riesgo & & 1 & $\oplus \oplus \# \mathrm{OO}$ & \\
\hline \multirow[t]{3}{*}{ VIH } & Población general con factores de riesgo & \multirow{3}{*}{ Serología } & $\mathrm{A}$ & $\mathrm{I} \oplus \oplus \oplus \oplus$ & \multirow{3}{*}{$7 / 2005$} \\
\hline & Población general sin factores de riesgo & & $\mathrm{C}$ & $-\oplus \oplus \# \mathrm{OO}$ & \\
\hline & Embarazadas & & A & $\mathrm{I} \oplus \oplus \oplus \oplus$ & \\
\hline \multirow[t]{2}{*}{ Herpes genital } & Embarazadas & \multirow[t]{2}{*}{ Serología } & $\mathrm{D}$ & $-\oplus \oplus \# \mathrm{OO}$ & \multirow[t]{2}{*}{$3 / 2005$} \\
\hline & Adolescentes y adultos & & D & $\mathrm{I} \oplus \oplus \oplus \oplus^{\#}$ & \\
\hline \multirow{4}{*}{$\begin{array}{l}\text { Infección por } \\
\text { Clamydia } \\
\text { trachomatis }\end{array}$} & $\begin{array}{l}\text { Mujeres sexualmente activas menores de } 25 \text { años, } 0 \text { en } \\
\text { mujeres mayores de } 25 \text { años con riesgo aumentado }\end{array}$ & \multirow{4}{*}{$\begin{array}{c}\text { Amplificación de ácidos } \\
\text { nucleicos en orina o } \\
\text { hisopado vaginal. }\end{array}$} & A & $\mathrm{I} \oplus \oplus \oplus \oplus$ & \multirow{4}{*}{$6 / 2007$} \\
\hline & $\begin{array}{l}\text { Mujeres embarazadas menores de } 25 \text { años, } 0 \text { en mujeres } \\
\text { embarazadas mayores de } 25 \text { años con riesgo aumentado }\end{array}$ & & B & $\mathrm{I} \oplus \oplus \oplus \mathrm{O}$ & \\
\hline & $\begin{array}{l}\text { Mujeres mayores de } 25 \text { años de bajo riesgo } \\
\text { (embarazadas o no) }\end{array}$ & & c & $-\oplus \oplus \oplus \mathrm{O}$ & \\
\hline & \begin{tabular}{|l|} 
Hombres \\
\end{tabular} & & I & $-\oplus \oplus \# \mathrm{OO}$ & \\
\hline
\end{tabular}

HBsAg: antígeno de superficie de la hepatitis B. VIH: infección por el virus de inmunodeficiencia humana.

Un * sobre el grado de recomendación indica que éste puede modificarse bajo ciertas circunstancias explicadas en el apartado correspondiente o por observaciones hechas por la CTFPHC

Grados de Evidencia: Buena (GRADE Alta $\oplus \oplus \oplus \oplus$ ) Aceptable (GRADE Moderada $\oplus \oplus \oplus \mathrm{O})$ e Insuficiente (GRADE Baja $\oplus \oplus$ OO / Muy Baja $\oplus$ OOO )

El \# indica que algunos componentes de la recomendación están basados también en un nivel inferior de evidencia. Por ejemplo $\oplus \oplus \#$ OO significa que una recomendación está basada en niveles de evidencia $\oplus \mathrm{OOO}$ y $\oplus \oplus \mathrm{OO}$

Nivel de recomendación: A Recomienda fuertemente el servicio (GRADE I: Fuerte); B: Recomienda el servicio (GRADE II: Débil); C Recomienda no proveer el servicio de manera rutinaria (GRADE no se expide); D: Recomienda no proveer el servicio (GRADE I: Fuerte); I: No puede hacerse una recomendación por evidencia insuficiente (GRADE no se expide). 


\section{Sífilis, en embarazadas y personas de alto riesgo (A)}

No se encontró evidencia directa nueva que indique que el rastreo de infección sifilítica mejore los resultados de salud en las personas de alto riesgo (hombres que tienen sexo no protegido con hombres, trabajadores del sexo, personas que intercambian sexo por drogas, adultos en correccionales o cárceles, y posiblemente personas con otras ETS). Sin embargo existe evidencia adecuada de que las pruebas de rastreo no treponémicas (VDRL o RPR), seguidas de la confirmación con pruebas treponémicas (FTA-ABS o TP-PA) pueden detectar la infección con precisión y que los antibióticos pueden curarla. Se desconoce el intervalo de rastreo óptimo en personas de alto riesgo. Si bien el rastreo puede producir daños potenciales (como la evaluación clínica de resultados falso-positivos, ansiedad innecesaria y daños por el uso de antibióticos), los beneficios de rastrear a personas de alto riesgo superan a los daños potenciales.

Por otro lado, existe evidencia observacional de que el rastreo universal en embarazadas en su primer control prenatal reduce la proporción de niños con manifestaciones clínicas de infección sifilítica y/o serologías positivas. Los beneficios de rastrear infección sifilítica a mujeres embarazadas superan substancialmente a los daños potenciales.

\section{Sífilis, en personas de bajo riesgo (D)}

Dado la baja incidencia de infección sifilítica en la población general, y el consecuente bajo rédito diagnóstico del rastreo, se concluye que el daño potencial asociado al mismo (costo de oportunidad, pruebas falso-positivas y etiquetamiento) supera a los beneficios.

\section{Gonorrea, en mujeres de alto riesgo (B)}

La infección asintomática por $\mathrm{N}$. gonorrhoeae puede ocasionar una elevada morbilidad relacionada con episodios de enfermedad pelviana inflamatoria (EPI), embarazo ectópico y dolor pelviano crónico. En las mujeres embarazadas, puede ocasionar corioamnionitis, ruptura prematura de membranas y parto pretérmino. Se consideran de alto riesgo a las trabajadoras del sexo, mujeres con episodios repetidos de gonorrea, uso irregular del preservativo, uso de drogas, nueva o múltiples parejas sexuales y posiblemente mujeres sexualmente activas menores de 25 años.

Existe evidencia suficiente de que el rastreo puede detectar con precisión la infección, y de que el tratamiento es efectivo. Las técnicas tradicionales de rastreo incluyen el examen directo y el cultivo de materia tomado a través de hisopado cervical y uretral; sin embargo existen técnicas más modernas con similares o mejores características operativas y mayor practicidad (amplificación e hibridación de ácidos nucleicos a partir de muestras en orina e hisopados vaginales, cervicales o uretrales).

Si bien no existe información que cuantifique los daños potenciales del rastreo (resultados falsos positivos, ansiedad y tratamientos antibióticos innecesarios), se estima que estos son pequeños.

Por lo expuesto, se recomienda el rastreo de Neisseria gonorrhoeae en mujeres asintomáticas con alto riesgo de infección (embarazadas o no) preferentemente mediante cultivo endocervical.

Gonorrea, en embarazadas de bajo riesgo u hombres de alto riesgo (I)*

En las embarazadas con bajo riesgo de infección por N. gonorrhoeae, la infección asintomática es poco frecuente. En tanto que en los varones con alto riesgo de infección la morbilidad asociada resulta menor que en las mujeres, probablemente porque la enfermedad asintomática es menos frecuente. La evidencia resulta insuficiente como para recomendar el rastreo a toda embarazada de bajo riesgo o a los hombres asintomáticos de alto riesgo.

De acuerdo a las recomendaciones de la CTF, en la población de alto riesgo (individuos menores de 30 años, particularmente adolescentes, con al menos dos parejas en el último año, o menores de 16 años de edad al inicio de sus relaciones sexuales, o contactos sexuales de individuos con una ETS) -incluidos los varones-, el rastreo es altamente recomendable $(A)$.

\section{Gonorrea, en población general adulta (D)}

Dado que la prevalencia de infección por N. gonorrhoeae es baja en la población general, se asume que en este escenario los perjuicios del rastreo superan sus beneficios.

Hepatitis B, en embarazadas en su primer control prenatal (A) Existe buena evidencia de que el rastreo prenatal de hepatitis $\mathrm{B}$, mediante $\mathrm{HBsAg}$, reduce su transmisión prenatal y el desarrollo de infección crónica en el recién nacido (dadas la posterior vacunación y profilaxis post-exposición con gamaglobulina hiperinmune específica a los neonatos de madres infectadas). Se recomienda el rastreo en el primer control prenatal. Se concluye que los beneficios del rastreo superan sustancialmente a los daños.

\section{Hepatitis B, en población general (D)}

La prevalencia de hepatitis $B$ en población general es baja. Por otro lado, la mayoría de los infectados no progresan a la cronicidad, cirrosis o hepatopatía. Esto, sumado al hecho de que no existe evidencia de que su rastreo en este grupo mejore los resultados a largo plazo, y teniendo en cuenta además los daños potenciales del rastreo (como el etiquetamiento), lleva a concluir que los daños del rastreo probablemente superen a los beneficios.

\section{Hepatitis C, en población general (D) y en adultos de alto riesgo (I)}

Existe buena evidencia de que el rastreo puede detectar la infección crónica por virus $\mathrm{C}$. Sin embargo, la prevalencia de esta infección es baja en la población general; y aunque podría ser mayor en individuos de alto riesgo (adictos intravenosos, receptores de hemoderivados antes de 1990, sometidos a diálisis, hijos de madres con hepatitis $C$, con conducta sexual de alto riesgo, o uso de drogas ilegales) la mayoría de los infectados no desarrollan cirrosis o resultados adversos mayores (evidencia limitada indica que 10 al $20 \%$ de los pacientes con infección crónica desarrollan cirrosis a los 20 a 30 años). Por otro lado, no existe evidencia de que el rastreo de hepatitis $C$ mejore los resultados clínicamente significativos a largo plazo, como la cirrosis, el carcinoma hepatocelular o la mortalidad específica. $Y$ aunque existe buena evidencia de que el tratamiento antiviral mejora resultados intermedios, como la reducción de la carga viral por virus $\mathrm{C}$, la evidencia de estos resultados a largo plazo es escasa, el tratamiento es prolongado, costoso y muchos pacientes lo abandonan por efectos adversos.

Entre los daños potenciales del rastreo se incluyen la realización de biopsias innecesarias y el etiquetamiento, sin embargo la evidencia en cuanto a su magnitud es limitada. 
Por lo expuesto se concluye que en la población general los daños potenciales del rastreo probablemente excedan los beneficios; mientras que, en la población de alto riesgo, no puede establecerse un balance neto a favor o en contra del rastreo.

\begin{abstract}
Infección por virus de inmunodeficiencia humana (VIH), en adolescentes y adultos con riesgo aumentado (A)

Se consideran individuos con riesgo aumentado a aquellos hombres que han tenido relaciones sexuales con hombres (a partir de 1975), a los hombres y las mujeres que tienen relaciones sexuales sin protección con parejas múltiples, a los usuarios de drogas endovenosas en el pasado o presente, a los hombres y mujeres que intercambian sexo por dinero o drogas, o que tienen parejas sexuales que lo hacen, a las personas cuyos parejas sexuales presentes o pasadas están infectadas por el VIH, a los individuos bisexuales, a personas en tratamiento por enfermedades de transmisión sexual, y las personas que recibieron transfusiones sanguíneas entre 1978 y 1985. Las personas que solicitan la prueba del VIH, aunque no reporten factores de riesgo individuales, también pueden ser consideradas de mayor riesgo, ya que este grupo puede incluir a personas que no están dispuestos a revelar los comportamientos de alto riesgo.
\end{abstract}

Existe buena evidencia de que las pruebas actualmente en uso detectan la infección por VIH con precisión, y que su tratamiento oportuno reduce la progresión de la enfermedad y su mortalidad específica. Por otro lado los resultados falsos positivos del rastreo resultan raros en esta población y los daños asociados al mismo (ansiedad, etiquetamiento y efectos en las personas cercanas) se consideran mínimos. La mayoría de los efectos adversos del tratamiento pueden ser corregidos modificando el esquema terapéutico o mediante un tratamiento apropiado.

Por lo expuesto se concluye que los beneficios asociados al rastreo superan ampliamente sus riesgos.

VIH, en adolescentes y adultos de bajo riesgo (C)

Si bien existe evidencia aceptable de que el rastreo de infección por VIH en individuos con bajo riesgo puede detectar casos adicionales, se estima que el rédito global de esta intervención sería bajo en una población con menor prevalencia de infección. En este escenario, comenzarían a pesar un poco más los efectos indeseables del rastreo (ver antes), por lo cual por el momento no puede establecerse una recomendación a favor o en contra del rastreo en esta población.

\section{VIH, en embarazadas (A)}

Existe buena evidencia de que las pruebas actualmente en uso detectan con precisión la infección por VIH en embarazadas, y que el consejo prenatal universal, seguido del rastreo voluntario, incrementa el número de mujeres diagnosticadas $\mathrm{y}$ tratadas antes del parto. También existe buena evidencia de que los tratamientos antirretrovirales son aceptados por las embarazadas y de que reducen significativamente la tasa de transmisión madre-hijo, sin evidencias de anomalías o problemas materno-fetales (con la excepción del tratamiento con efavirenz).

La detección temprana permite discutir la indicación de cesárea electiva y evitar la lactancia materna para reducir la tasa de transmisión de VIH por esta vía.

Los beneficios de rastrear a toda mujer embarazada superan sustancialmente los daños potenciales.
Herpes genital, en embarazadas (D)

Existe evidencia aceptable de que el rastreo serológico del virus herpes simplex (VHS) en embarazadas asintomáticas no reduce su transmisión neonatal. Son las mujeres que desarroIlan infección primaria por VHS quienes poseen el mayor riesgo de transmisión de la infección a sus hijos. Sin embrago, dado que estas mujeres resultan inicialmente seronegativas, el rastreo serológico del VHS (mediante ELISA o Western blot) no detecta con precisión a las mujeres de mayor riesgo. Por otro lado, no se encontró evidencia de que tratar a las mujeres seronegativas reduzca el riesgo de infección neonatal; y la evidencia es limitada en cuanto a que el tratamiento antiviral en mujeres con herpes genital recurrente, o la indicación de cesárea en mujeres con lesiones activas por VHS al momento del parto, reduzcan el resultado de interés. También resulta limitada la evidencia de la seguridad de la terapia antiviral en embarazadas y neonatos.

Los daños potenciales del rastreo, aunque no bien estudiados, incluyen resultados falsos positivos, etiquetamiento, ansiedad, así como falsos negativos y falso reaseguro.

Se concluyó que no hay beneficios asociados al rastreo por lo que los daños potenciales superan los beneficios.

\section{Herpes genital, en adolescentes y adultos (D)}

Si bien existe buena evidencia de que la serología puede identificar con exactitud a las personas expuestas al VHS y que el tratamiento antiviral mejora algunos resultados en salud en personas sintomáticas (por ej. las recurrencias frecuentes), no se halló evidencia de que el rastreo en adolescentes y adultos asintomáticos mediante serología mejore los resultados en salud, los síntomas o la transmisión de la enfermedad. Los daños potenciales del rastreo incluyen resultados falsos positivos, etiquetamiento y ansiedad, aunque su evidencia es limitada.

Se concluyó que los beneficios del rastreo son, en el mejor de los casos, mínimos y que los daños potenciales superan los beneficios.

Infección por Clamidia trachomatis (IC), en mujeres sexualmente activas menores de 25 años, o en mujeres mayores de 25 años con riesgo aumentado (A)

La infección asintomática por Clamidia trachomatis puede ocasionar una elevada morbilidad relacionada con episodios de uretritis, cervicitis, EPI, dolor pelviano crónico, embarazo ectópico o infertilidad. Se considera con riesgo incrementado de IC a todas las mujeres sexualmente activas menores de 25 años (incluidas las adolescentes), con antecedentes de IC u otra ETS, con una nueva pareja sexual o múltiples parejas, que realicen un uso irregular de preservativo o intercambien sexo por drogas o dinero.

Existe buena evidencia de que el rastreo en este grupo puede reducir la incidencia de EPI y evidencia aceptable de que puede reducir la prevalencia de IC. Las pruebas de amplificación de ácidos nucleicos son las más recomendadas actualmente para el rastreo de Clamidia, ya que son más sensibles, específicas y de mayor practicidad que las pruebas tradicionales (aunque en poblaciones de baja prevalencia la tasa de falsos positivos aumenta). No se conoce el intervalo óptimo del rastreo. En mujeres con un resultado previo positivo se recomienda repetirlo en seis a 12 meses dada la alta tasa de reinfección; mientras que en el caso de un resultado previo negativo no seria necesario rastrear tan frecuentemente. Las parejas de individuos infectados también deben ser identificadas y tratadas empíricamente, o en base al resultado de las 
pruebas diagnósticas. Se estima que los daños del rastreo son pequeños, fundamentalmente relacionados a los resultados falsos positivos y el sobretratamiento. Se concluye que los beneficios asociados al rastreo en este grupo superan sustancialmente los daños potenciales.

Infección por Clamidia trachomatis, en mujeres embarazadas menores de 25 años, o en mujeres embarazadas mayores de 25 años con riesgo aumentado (B)

En las mujeres embarazadas la IC puede ocasionar aborto, corioamnionitis, ruptura prematura de membranas, parto pretérmino, bajo peso al nacer y aumento de la mortalidad neonatal. Existe aceptable evidencia de que el rastreo reduce la tasa de niños con bajo peso y prematurez. Aun no esta claro el momento óptimo de su implementación: al comienzo del embarazo mejora los resultados, pero hacia el tercer trimestre podría ser más efectivo en prevenir el contagio al niño durante el parto. Se desconoce el beneficio incremental de repetir el rastreo.Dado que hay moderada evidencia de que los benefi- cios son superiores a los daños se recomienda el rastreo de rutina en estos grupos de mujeres embarazadas.

Infección por Clamidia trachomatis, en mujeres mayores de 25 años de bajo riesgo, embarazadas o no (C)

Dado la baja prevalencia de la infección en este grupo se estima que el rédito diagnóstico del rastreo sería escaso. Sumado ésto al hecho de encontrarse evidencia moderada de que los beneficios son levemente superiores a los daños hace que no se pueda establecer una recomendación ni a favor ni en contra del rastreo en este grupo.

\section{Infección por Clamidia trachomatis, en hombres (I)}

Es probable que el beneficio directo del rastreo en los hombres asintomáticos sea pequeño, aunque el impacto del mismo podría ser mayor de apuntarse a una disminución en la incidencia de la IC en las mujeres. Aun así no se encontró evidencia que apoye este resultado y, por tanto, se concluyó que los beneficios del rastreo en hombres son desconocidos.

Recibido el 20/05/2009 y aceptado el 20/06/2009

\section{Referencias}

1. Ciapponi A. Guía de Práctica Clínica Actualizaciones de cuidados preventivos de la USPSTF (3 ra edición, 2000-2003) Disponible en: http://www.foroaps.org/hitalba-paginaarticulo.php?cod_producto=956

2. Ciapponi A. Nueva guía de cuidados preventivos de la Fuerza de Tareas de Estados Unidos (primera entrega). Evid actual pract ambul 2005;8:179-182. Disponible en: http://www.foroaps.org/files/nueva\%20fuerza.pdf

3. Adaptado por Ciapponi A de la. Nueva guía de cuidados preventivos de la Fuerza de Tareas de Estados Unidos (segunda entrega). Evid. actual. práct. ambul; 9(1): 26-30, Ene-Feb 2006. Disponible en: http://www.foroaps.org/files/cuida\%20preven\%202\%20parte.pdf

4. Ciapponi A. Actualización y combinación de las guías de cuidados preventivos de las fuerzas de tareas de EE.UU. y Canadá.(Primera parte: Consejería). Evid.Act. Pract. Ambul. 12(2). 61-65. Abr-Jun. 2009. Disponible en: http://www.foroaps.org/files/fb\%20gf\%20bgf.pdf 